\title{
Numerical modeling of deformation and vibrations in the construction of large-size fiberglass cooling tower fan
}

\author{
Shmakov Arthur Fanisovich, Modorskii Vladimir Yakovlevich \\ Federal State-Funded Educational Institution of Higher Professional Education Perm National Research Polytechnic University, Center of \\ high-performance computing systems, Perm, Russia (29 Komsomolsky prospekt, Perm - GSP, Perm krai, Russia, 614990)
}

\begin{abstract}
This paper presents the results of numerical modeling of deformation processes and the analysis of the fundamental frequencies of the construction of large-size fiberglass cooling tower fan. Obtain the components of the stress-strain state structure based on imported gas dynamic and thermal loads and the form of fundamental vibrations. The analysis of fundamental frequencies, the results of which have been proposed constructive solutions to reduce the probability of failure of the action of aeroelastic forces.
\end{abstract}

\section{Introduction}

In Russia, the cooling towers are about 3500 pieces. Search capabilities significantly (by more than by 2 times) reduction in energy consumption of electric fans cooling towers can be carried out in the direction of optimal aerodynamic profiling of working blades and the use of new materials. This paper presents the results of numerical modeling of the fan blades for cooling towers of large diameter from the point of view of oscillatory processes

Today one of the tools of modern engineering structures is a computer experiment. The global level of numerical studies in this field allows you to simulate the oscillatory processes in complex, large-scale structures with sufficient accuracy. Existing methods of designing cooling towers fans do not provide evaluation of oscillatory processes. However, there are currently no techniques, allowing to simulate the vibration of large cooling tower fans based gas dynamic loads. The work implemented stages of the modeling of stress-strain state of the large structure, taking into account gas-dynamic loads and analyzes the forced and natural frequencies. However, consideration of the above factors is a prerequisite for the correct description of vibrations of structural elements. Accounting gas dynamic and deformation processes even in the quasi-stationary formulation can significantly refine existing techniques and to predict the vibration in a large fiberglass fan, so in a timely manner, at the design stage, to take measures to prevent such occurrences. Furthermore, it will be possible, instead of the traditional use of materials for making the fan blades advanced composite materials based on glass fiber. Applications fiberglass material will allow to create effective aerodynamic blade design to reduce weight and energy usage of the drive motor and thus increase by 10 $20 \%$ or more efficiency of the cooling tower.

\section{Discussed problems}

The occurrence of vibrations in the cooling tower fans today can't be predicted. For certain values existing cooling tower vibrations increases the likelihood of failure of the fan and thus stop the production line. This can lead to significant losses of the enterprise.

To solve problems of vibration and oscillation of the blades, there are several methods. The first is to determine the natural frequencies of the structure and analysis of the response to harmonic load [1]. The result is a frequency and the natural vibration mode. This information will determine the operating frequency range of designs. Frequency method uses data from a stationary calculation of stress-strain state of the structure. This approach does not take into account the possibility of changes in the gasdynamic loads and, therefore, does not have enough accuracy.

The second method of estimating parameters of vibrations of the structure is to solve the problem aeroelastic [2]. In this case, problems are solved together gas-dynamic calculation and evaluation of the stress-strain state of the structure. This approach allows you to get a information as close as possible to reality and takes into account the changing load.

Interdisciplinary Problem Solving involves considerable computational complexity and to simplify the method of calculation of large fans cooling towers it is advisable to use the first frequency method, which is presented in this article.

\section{Physical model}


For the physical model was develop for studies describing oscillatory processes in large-sized fiberglass fan [3]:

- material fan blades adopted isotropic;

- field of gas-dynamic loads exported into account the stress-strain state of the construction and taken into account when calculating the natural frequencies;

- oscillatory processes in construction are considered in three-dimensional statement;

- considered the first three modes of natural oscillations, because the occurrence of resonance for them the most dangerous for the structure.

\section{Mathematical model}

Determination of the natural frequencies of vibrations of large-sized fan design, carried out using a mathematical model based on the use of Lagrange II-nd kind of differential equations [3]:

$$
\frac{d}{d t}\left(\frac{\partial T}{\partial \dot{x}_{l}}\right)-\frac{\partial T}{\partial x_{i}}=-\frac{\partial \Pi}{\partial x_{i}}-\frac{\partial \Phi}{\partial \dot{x}_{l}}
$$

where: $T$ - kinetic energy of the system, $\Pi$ - potential energy of the system, $i$-generalized coordinate, $x_{i}$, - the movement of the i-th element $(i=1,2,3), \Phi$-dissipative function, $t$ - time.

Kinetic energy of the system is:

$$
T=\sum_{i=1}^{3} T_{i}
$$

Potential energy of the system is:

$$
\Pi=\sum_{i=1}^{3} \Pi_{i}
$$

Dissipative function is proportional to the velocity of the center of mass:

$$
\begin{gathered}
\Phi=\sum_{i=1}^{3} \Phi_{i} \\
\Phi_{i}=\frac{-}{2} \beta_{i} \dot{x}_{i}
\end{gathered}
$$

where $\beta_{i}$ - damping coefficient.

\section{Algorithm of numerical modeling}

For the numerical calculations in the software package ANSYS Workbench engineering studies carried out on the basis of gas-dynamic calculations [4] was created by the geometric design and a model for strength and modal analysis (fig. 1). The computational model consists of four major elements: fan hubs, tubular longeron, fan tracery и blade plating. Due to the existing symmetry in the calculation is sufficient to consider only one blade.

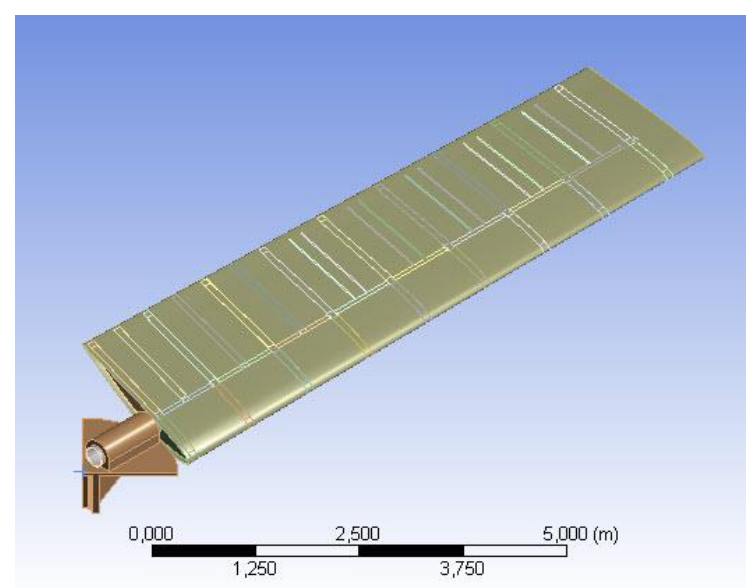

Figure 1. Calculated geometry of the large-sized fan blades.

Two variants of settlement construction large size fan blade has been designed For the frequency analysis. The construction of the first embodiment (Construction 1) It consists of a tubular longeron, fan tracery and blade plating. The material all elements of blades is fiberglass. This structure has a low weight and a high specific stiffness. But in this design there are high deflections at the edge of the blade, which may adversely affect the performance of the blade and adhesive joints.

Second Embodiment (Construction 2) is a reinforced structure. The tubular spar mounted steel tube for increased rigidity. To decrease the blade deflection center is supported by a cable.

On the basis of the calculation model is built finite element mesh (fig. 2). Time of construction of models of high performance computing complex PNRPU [5] after the debug computational geometry ranged from 20 to 60 minutes depending on the number of cells. The grid consisted of 216169 computational cells. To build a grid model used the built-in Ansys Workbench Meshing tool.

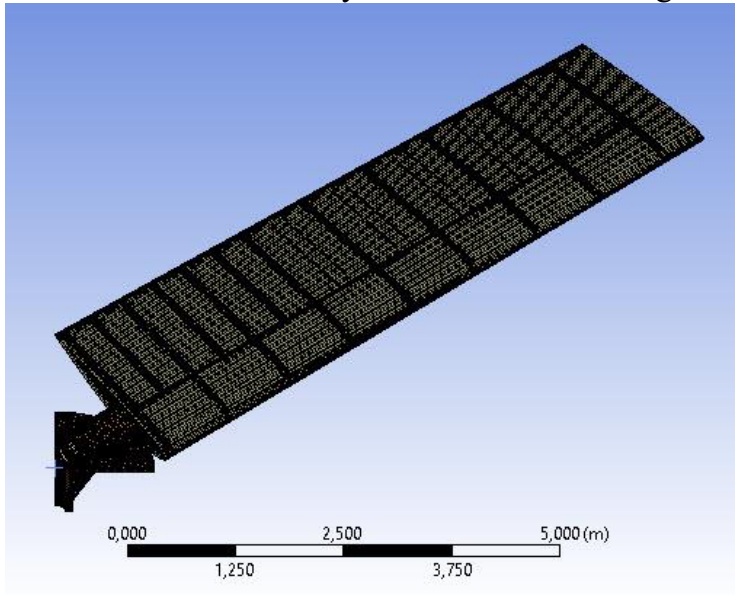

Figure 2. Finite element model large size fan blade.

To determine the natural frequencies (modal analysis) fan blade design took into account the action of aerodynamic, gravitational and inertial loads (fig. 3). As the boundary conditions set limits movement of the hub in place on the motor shaft (gear) and the imposition of conditions on the symmetry of the section of the border. 


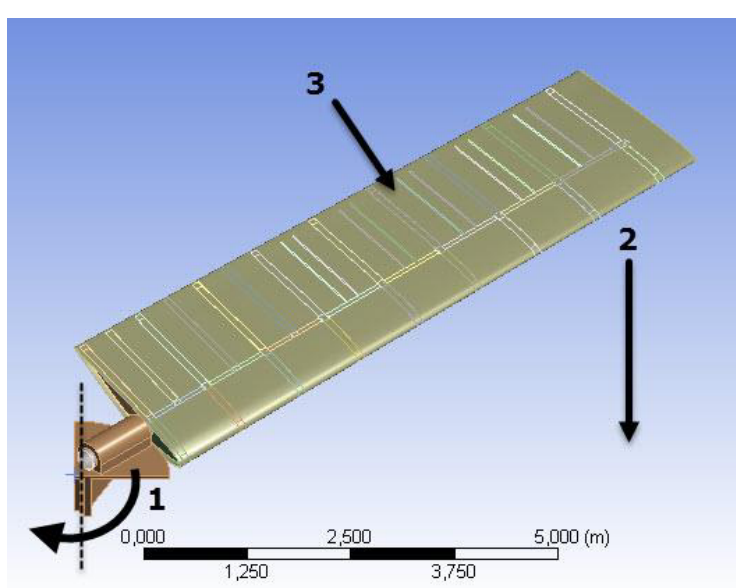

Figure 3. Loads for calculation the stress-strain and prestressed modal analysis: 1 - tower fan rotation direction; 2 - direction of action of the Earth's gravity; 3 - aerodynamic load application surface.

\section{Computational examples and analysis}

In Fig. 4 shows the first mode design large size fan blade vibrations. At a frequency of $1.99 \mathrm{~Hz}$ natural oscillations observed in the plane of rotation of the fan. This frequency is close to the maximum operating speed of the fan, which can lead to destruction of the blade and resonance. In the second mode at a frequency of $3.23 \mathrm{~Hz}$ observed natural oscillations in the vertical plane. This plane may experience vibrations caused by aerodynamic and gravitational forces. You may experience a so-called flutter - self-excited undamped self-oscillations.

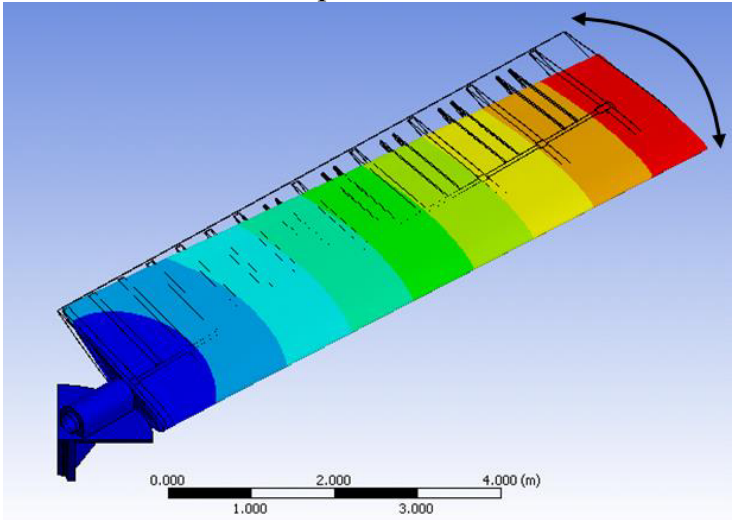

a)

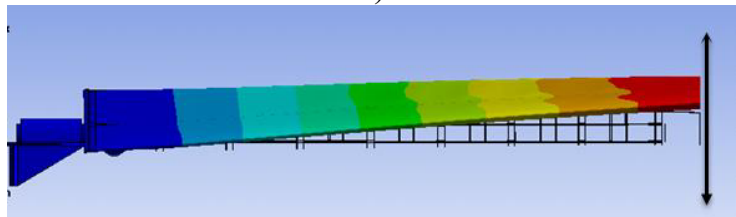

b)

Figure 4. Modes of the natural oscillations large size cooling tower fan blades at a frequency of $1.99 \mathrm{~Hz}$ (a) and $3.23 \mathrm{~Hz}$ (b).

For a more accurate analysis of large size fan cooling tower built resonance diagram for the the first two or three modes of oscillations in the operating speed range (fig. 5). These modes of distribution of energy as much as possible and in the event of resonance can be destroyed blades. As can be seen from the diagram, there are first intersection line with the natural frequency of the second harmonic and higher. The points of intersection are in the operating range, which is critical to the efficiency of the cooling tower fan.

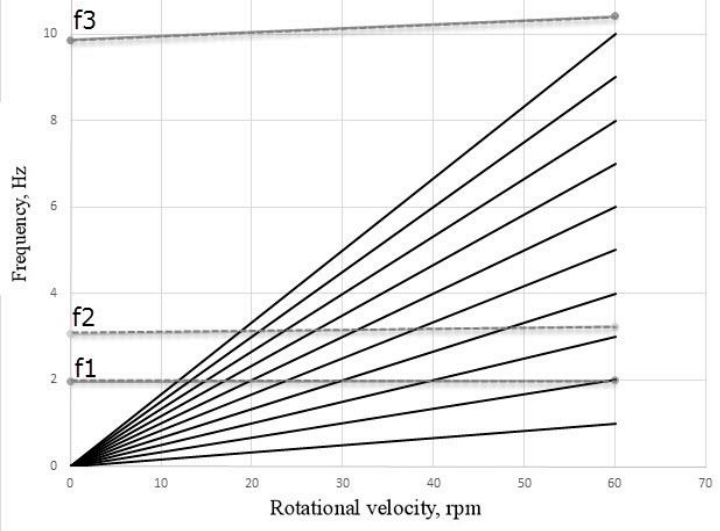

Figure 5. Resonance diagram large size fan blades cooling tower for Constructions 1 .

Frequency analysis for the blade structure 2 was carried out to increase the natural frequency and resonance diagram built (fig. 6). As can be seen from the diagram, there are the intersection of the first natural frequency of the line with the ninth and higher harmonics. These harmonics are safe for the fan performance.

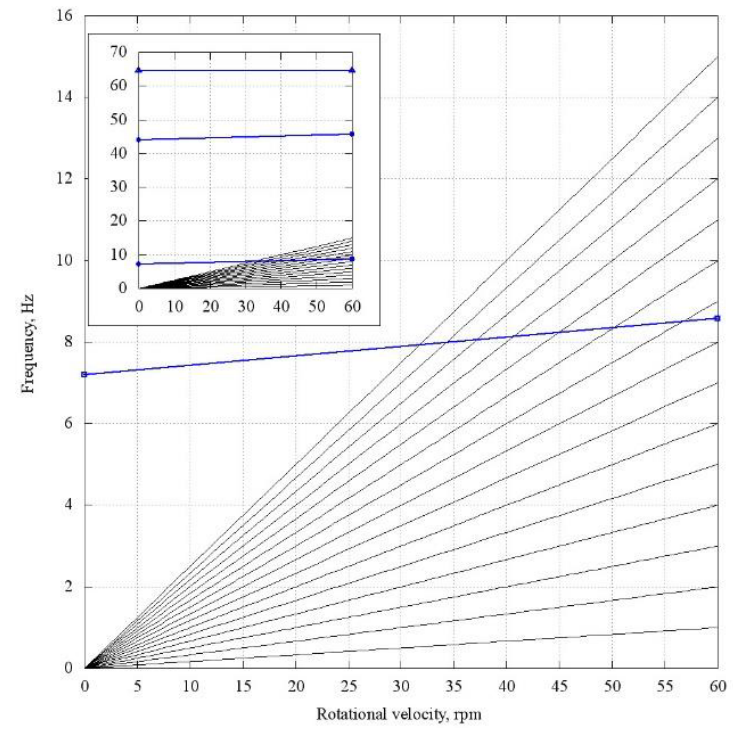

Figure 6. Resonance diagram large size fan blades cooling tower for Constructions 2.

According to calculations it was made blade Construction 2. For the manufacture of the blade surface on the engraving and milling machine of «Center for Innovative Engineering Technologies» PNRPU on a reported basis master model of the blade section have been prepared. After you create a master model was fabricated form for displaying the shell portion of the blade. Power blade construction consists of a tubular carrier longeron and ribs mounted thereon. At the final stage the assembly of the blade casing and the power structure. Manufactured the blade is shown in fig 7, a. After manufacture of the blades and their connection to the hub of the fan 
installation occurs directly on the tower (fig 7, b). During the pilot operation of the sample experienced fan following characteristics achieved: Power consumption was less than $200 \mathrm{~kW}$, instead of project $450 \mathrm{~kW}$; temperature difference cooling water tower rose from $4{ }^{\circ} \mathrm{C}$ using a precursor to an analogue to $8^{\circ} \mathrm{C}$, on the prototype.

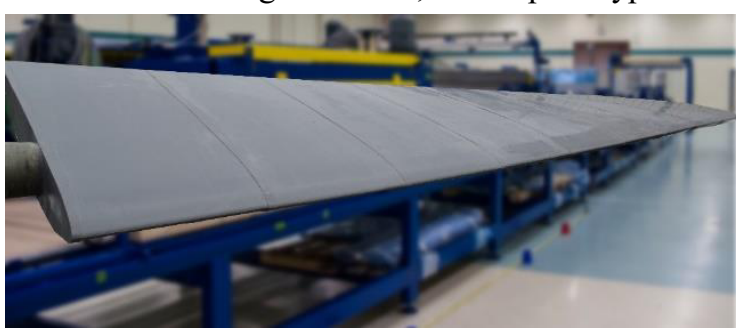

a)

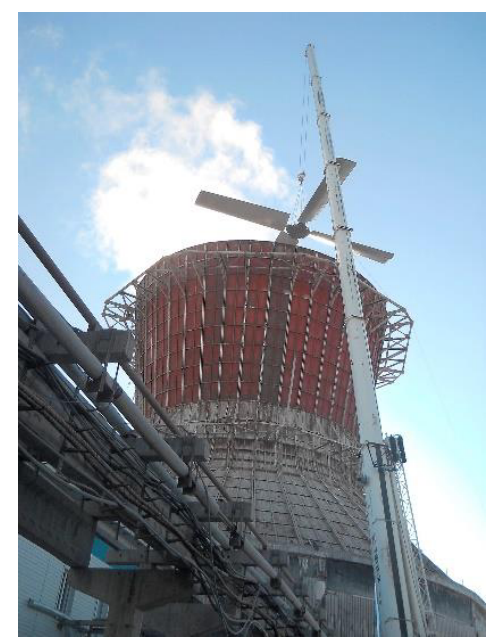

b)

Figure 7. Assembly (a) and installation (b) of large size fan in the cooling tower.

\section{Conclusions}

As a result of this work the following conclusions:

1. To eliminate the resonance effects in the design of the design large size tower fan resonance diagrams necessary to calculate and ensure the absence of oscillations in the operating frequency range.

2. The method of numerical experiments allow to consider gas dynamics and gravitational load when calculating the vibration large size fan design elements has been developed.
3. On the basis of the developed methodology as a result of numerical simulation of the blade design large size tower fan has been designed, which provides the necessary strength, consumables and energy characteristics.

\section{Acknowledgements}

The research work was supported by Russian Science Foundation under Grant No. №14-19-00877.

\section{References}

1. A. Shmakov, V. Modorskii, Modeling of oscillating processes in elements construction SRM, Scientific and Technical Volga region Bulletin, 6, 368-371 (2013)

2. E. Mekhonoshina, V. Modorskii, On a phase-shift of waves at the medium interface, Computer Optics, 39(3), 385 (2015).

3. A. Klepikovsky, Y. Timofeev, A. Shaiko-Shaikovsky, The refined mathematical models of estimation natural frequency systems with several degrees of freedom, Nika (2011) (URL: http://cyberleninka.ru/article/n/utochnyonnayamatematicheskaya-model-otsenki-sobstvennyhchastot-kolebaniy-sistem-s-neskolkimi-stepenyamisvobody)

4. A. Shmakov, V. Modorskii. Energy Conservation in Cooling Systems at Metallurgical Plants, Metallurgist, 59(9), 882-886 (2016)

5. A. Shmakov, V. Modorskii. Numerical Modeling of Gasdynamic Processes and Processes of Deformation in Compressor of Model Test Bench of the GasDistributing Unit, AMM, 799-800, 865-869 (2015)

6. M. Dimentberga, S. Kolesnikov, Vibrations in thechnics. Vibrations machines, structures and their elements (Moscow, Mashinostroyeniye, 1980)

7. V. Modorskii and other, The solution of engineering problem on the high-performance computer system of the Perm national research polytechnical university: monograph. (Perm national research polytechnical university, 2014) 